\title{
MASKING OF SHORT PROBE SOUNDS BY TONE BURSTS WITH A SWEEPING FREQUENCY *
}

\author{
GUIDO F. SMOORENBURG $a$ and FRANS CONINX $b, \cdots *$ \\ a Institute for Perception TNO, Soesterberg, and b Department of Medical and Physiological Physics, \\ State University of Utrecht, The Netherlands
}

(Received 22 April 1980; accepted 24 September 1980)

The masked threshold of short $(<40 \mathrm{~ms})$ probe tone or a short one-third octave probe noise appears to increase if the frequency of a tonal masker is swept. Frequency sweeps were exponential (xctaves/s) and unidirectional. Probe sounds were presented in the time center of the masker at the inter frequency of the masker. The sweep speed, $S$, appeared to be an inportant parameter; masker curation was much less important. For $10-\mathrm{ms}$ tonal probes, in-phase with the masker in their common time center, 100-ms maskers, and upward sweeps, increase of the masked threshold appeared to be maximal at a sweep speed of $30 \mathrm{cct} / \mathrm{s}$ and the masked threshold was $21 \mathrm{kM}$ higher than the masked threshold found for the stationary masker $(S=0 \mathrm{oct} / \mathrm{s})$. Above $30 \mathrm{oct} / \mathrm{s}$ the masked threshold decreased. For downward sweeps masking was maximal at $S=20 \mathrm{oct} / \mathrm{s}$ and the threshold was $15 \mathrm{~dB}$ higher. Sweeping upward the increase in masking was $12 \mathrm{~dB}$ for both noise probes and tonal probes with phase differing by $90^{\circ}$ from the masker in their conmon time center. The results are inconsistent with current models of masking: sweeping the frequency the masked threshold increases whereas the energy within the critical band at the probe frequency decreases.

Key words: masking, short probe sound, tone bursts, sweeping frequency.

\section{INTRODICTION}

In this paper we describe some experiments an masiking of a short probe sound by a tone burst with a sweeping frequency. Up to certain sweep speeds, masked thresholds appear to be higher than the thresholds found for stationary riaskers. This is in contradiction with current masking theories based on energy detection in critical bands.

In a previous paper [2] we reported that the loudness of a $50 \mathrm{~ms}$ tone burst increases when its frequency is swept. For staticnary stimuli it was well known that wide-band stimuli are louder than narrow-band stimuli at the same total sound pressure level (SPL). Although the SPL per critical band is less fur wide-band stimuli a higher loudness is found because of the way in which loudness summates over critical bands. For a detailed review of the critical band concept the reader is referred to Scharf $[9,10]$. Our results suggested that it makes little difference for lcidness perception whether the frequency compor,ents

\footnotetext{
* Part of this study was reported before at the 98th meeting of the Acoustical Society of America in Salt Like City, November 1979 [3].

** Present address: Institute for the Deaf, St. Michielsgestel, The Netherlands.
} 
are presented sim sltaneously or whether we sweep through the frequency range within a short period of tirne. The loudness data were closely matcheil by predictions based on the loudness calculation method of Zwicker and Feldtkeller [14,15] taking into account the frequency range covered by the sweep but disregarding the temporal aspect of the sweep. Thus, the increase in loudness could be understood on the basis of a well known effect of bardwidth on loudness and the additional assumption of an integration mechanism for loudness with a time constant of about $50 \mathrm{~ms}$ or more.

In contrast to the effect on loudness, an increase in masked threshold for a sweeping frequency cannot be understood on the basis of current masking theories. According to these theories (e.g. [15]) the masked threshold is determined by detection, in some critical band of an energy increment introduced by adding the probe to the masker. If probe and masker are single-frequency stimuli at the same frequency the energy increment will be detected in the critical band tuned to this frequency. (Here we disregard the possibility of off-frequency detection which does not affect the reasuning in principle.) The energy supplied by the masker to the critical band tuned to the probe will decrease when the masker is swept and, hence, a decrease of the masked threshold is expected.

The reasoning given above is somewhat simplified since phase effects were not taken into account. Sweeping the frequency results in a running phase relation between masker and probe. The energy increment depends on the phase relation between masker and probe. It is maximal if they are in-phase $\left(0^{\circ}\right.$ phase difference) and it is minimal if their phases differ by $90^{\circ}$. Thus, when we start with an in-phase condition and next the masker is swept, the energy increment may decrease because the phase relation between masker and probe shifts with time. The decreasing energy increment implies an increase in nasked threshoid. Of course, the effect of the running phase is limited by the probe sound duration and by the critical bandwidth. Since the energy increment is minimal for a $90^{\circ}$ phase difference no increase in masked threshold is expected from the running phasc reiation when we stari with this phase difference. The effect of phase was tested stparately in an experiment with initial phase differences of both $0^{\circ}$ and $90^{\circ}$. The main body of experiments was run with either an initial phase difference of $0^{\circ}$ or a probe sound consisting of a narrow band of noise resulting in a random phase relation.

The experiments were done in two parts. In the experiments that were carried out first, but which are presented here second (Part B), the probe was a 10 ms burst of $1 / 3$. octave noise. These experiments were performed in the Department of Medical and Physiological Physics, State University of Utrecht [1]. Four young men, trained extensively in the task, participated in these experiments. In the experiments that were carried out second, but which are presented here first (Part A), a tonal probe was used. These experiments weri performed in the Institute for Perception TNO at Soesterberg with nine subjects who had general experience in psychoacoustic experiments but were not trained in this tinsk and one subject (FC) who particpated also in the first experiments.

\section{A. TONAL PROBE}

\section{Stimuli}

The masker was a tone burst with a single frequency component that was swept upward or downward. The stimuli, masker and probe, are schematized in Fig. 1. Masker 


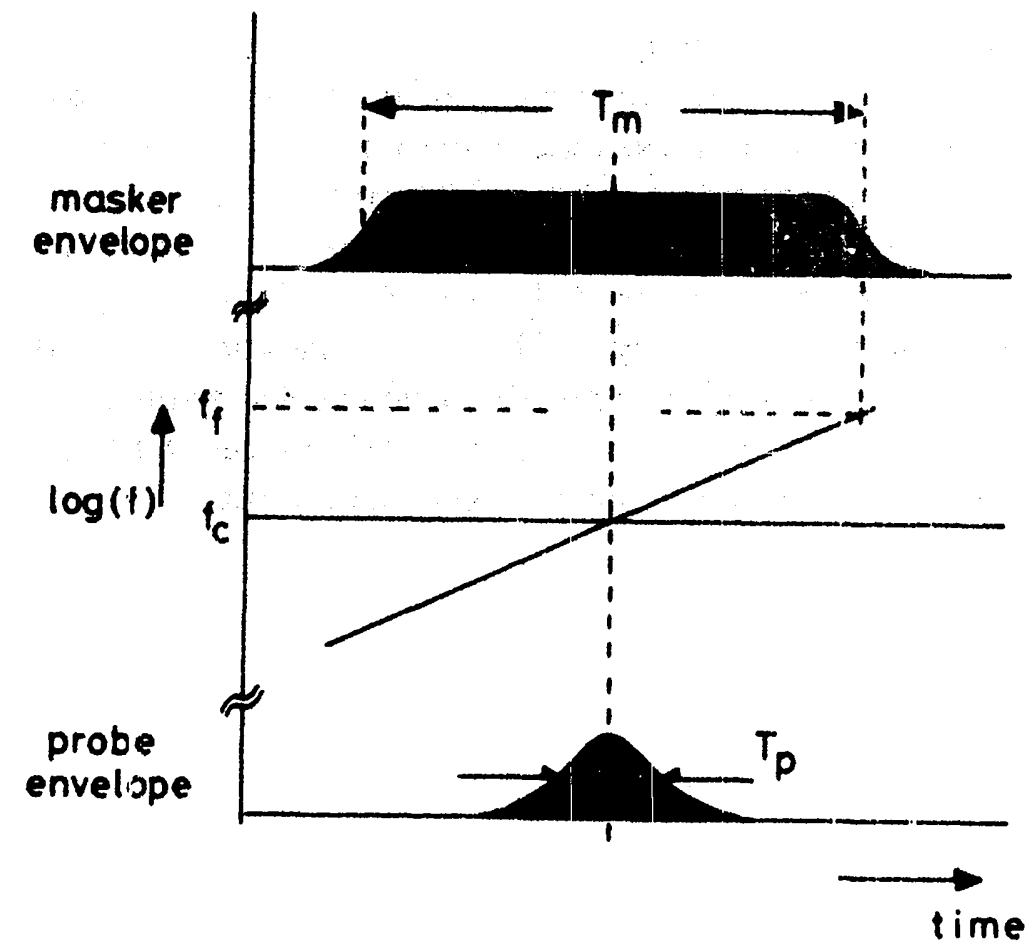

Fig. 1. Stimuius configuration. The upper part shows the masker amplitude, the middle part the masker frequency and the lower part the probe amplitude as a function of time.

onset and offset were cosine-shaped to reduce audible switching clicks. Masker duration was $100 \mathrm{~ms}$, onset and offset times were $10 \mathrm{~ms}$. The frequency of the masker in its time center, $f_{0}$, was equal to $1000 \mathrm{~Hz}$ for all sweep speeds. Frequency was swept exponentially with time so that $\mathrm{lcg} f$ was swept linearly. The exponential sweep was chosen in view of logarithmic frequency coding by the ear for most of the frequency range. It

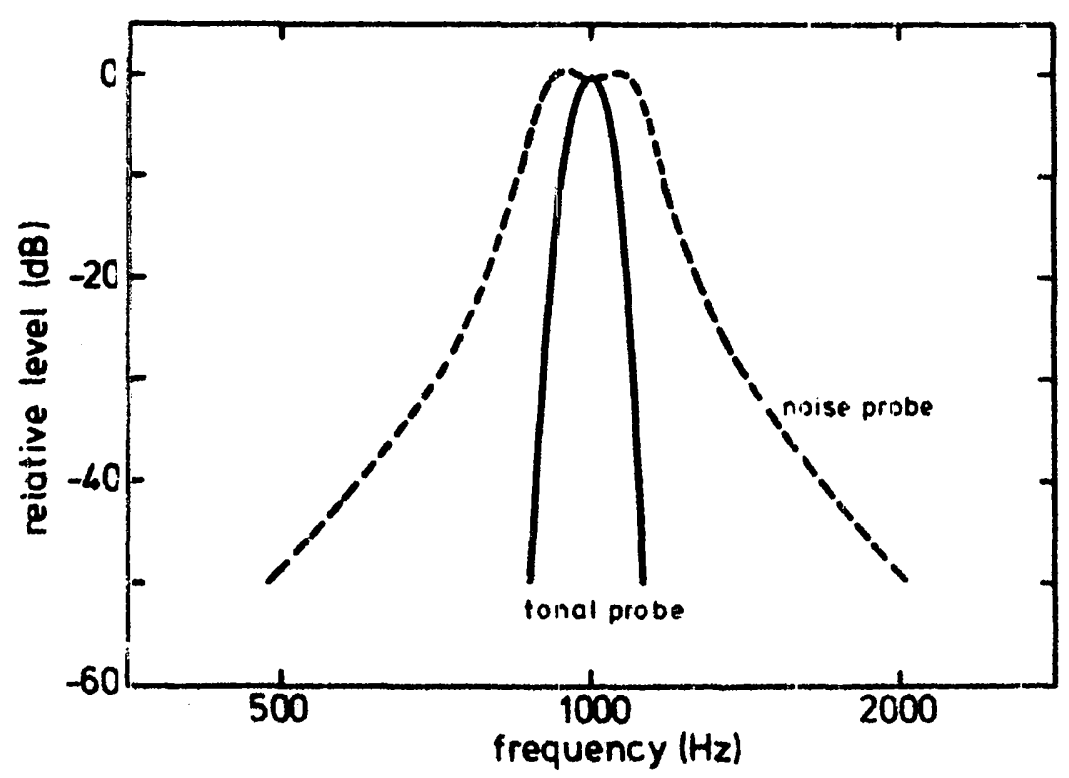

Fig. 2. Spectra of the $10 \mathrm{~ms}$ Gaussiarl-shaped tonal probe used in the experiments of Part $A$ and of the noise probe used in Part B. 
implies a sweep speed, $S$, in octaves per second (cct/s).

The probe was a $1000 \mathrm{~Hz}$ signal presented in the time center of the masker. The phase difference between probe and masker in the common time center was fixed. The temporal envelope of the probe was Gaussian-shaped with a duration of $10 \mathrm{~ms}$ as defined by the full-width at half-maximum. This implies a bandwidth at the $-3 \mathrm{~dB}$ level of about $70 \mathrm{~Hz}$. The spectrum of this probe and that of the noise probe used in part B are shown in Fig. 2.

Both masker and probe signals were produced by a DEC PDP-1110 computer and peripheral revolving memories. The probe signal, including the envelope, wass synthetized by 256 samples of 10 bits and $125 \mu \mathrm{s}$ sample time, the masker by 3600 samples of 10 bits and $35 \mu \mathrm{s}$ sample time. The masker signal $M(t)$ was computed using the formuia

$M(t)=\mathrm{A} \cdot \cos \left\{\frac{2 \pi}{S \cdot \ln 2} \cdot f_{\mathrm{c}} \cdot\left(2^{S t}-1\right)\right\}$

The instantaneous frequency $f(t)$ follows from differentiation with respect to time of the cosize-argument:

$f(t)=\frac{\mathrm{d}}{\mathrm{d} t}\left\{\frac{1}{S \cdot \ln 2} \cdot f_{\mathrm{c}} \cdot\left(2^{S t}-1\right)=f_{\mathrm{c}} \cdot 2^{S t}\right\}$

$f_{\mathfrak{c}}$ is center frequency, $S$ is sweep speed and $t$ is time. The masker signa was low-pass filtered at $14 \mathrm{kHz}$, and the probe at $4 \mathrm{kHz}$. Via computer-controlled attenuators they were fed into a mixing network which was connected with a Beyer DT 48 headphone.

\section{Methods}

Masked thresholds were measured with a self-paced two-alternative forced-choice paradigm. Immediately upon the response, the subject received visual information about its correctness. An adaptive procedure was used, starting with a probe level at which the probe was clearly audible for all sweep speeds. After 5 trials at this level, the probe level was decreasell by $2 \mathrm{~dB}$ after each correct response and increased by $6 \mathrm{~dB}$ after each incorrect response. The threshold was computed from the resulting up-and-down sequence of the probe level by averaging 50 probe levels after the first reversal in the upand-down sequence. The procedure converges to an average probe level corresponding to $75 \%$ correct response probability. (The a-priori probability of a correct response, guessing, was 50\%.) For each condition the masked threshold was measured twice. Each threshold measurement took about 3 to $4 \mathrm{~min}$. The computer which produced masker and probe signals was also used to control the adaptive measuring procedure and to compute the threshold values.

\section{Results}

Masked thresholds for upward and downward sweeps

Masked thresholis were measured as a function of sweep speed from $S=0$ oct/s (stationary masker) up to $S=80 \mathrm{oct} / \mathrm{s}$. At the maximum speed of $80 \mathrm{oct} / \mathrm{s}$ the frequency of the masker, $100 \mathrm{~ms}$ in duration, moved within the range from $62.5 \mathrm{~Hz}$ to $16 \mathrm{kHz}$ with the center frequency at $1 \mathrm{kHz}$. In the common time center probe and masker were 

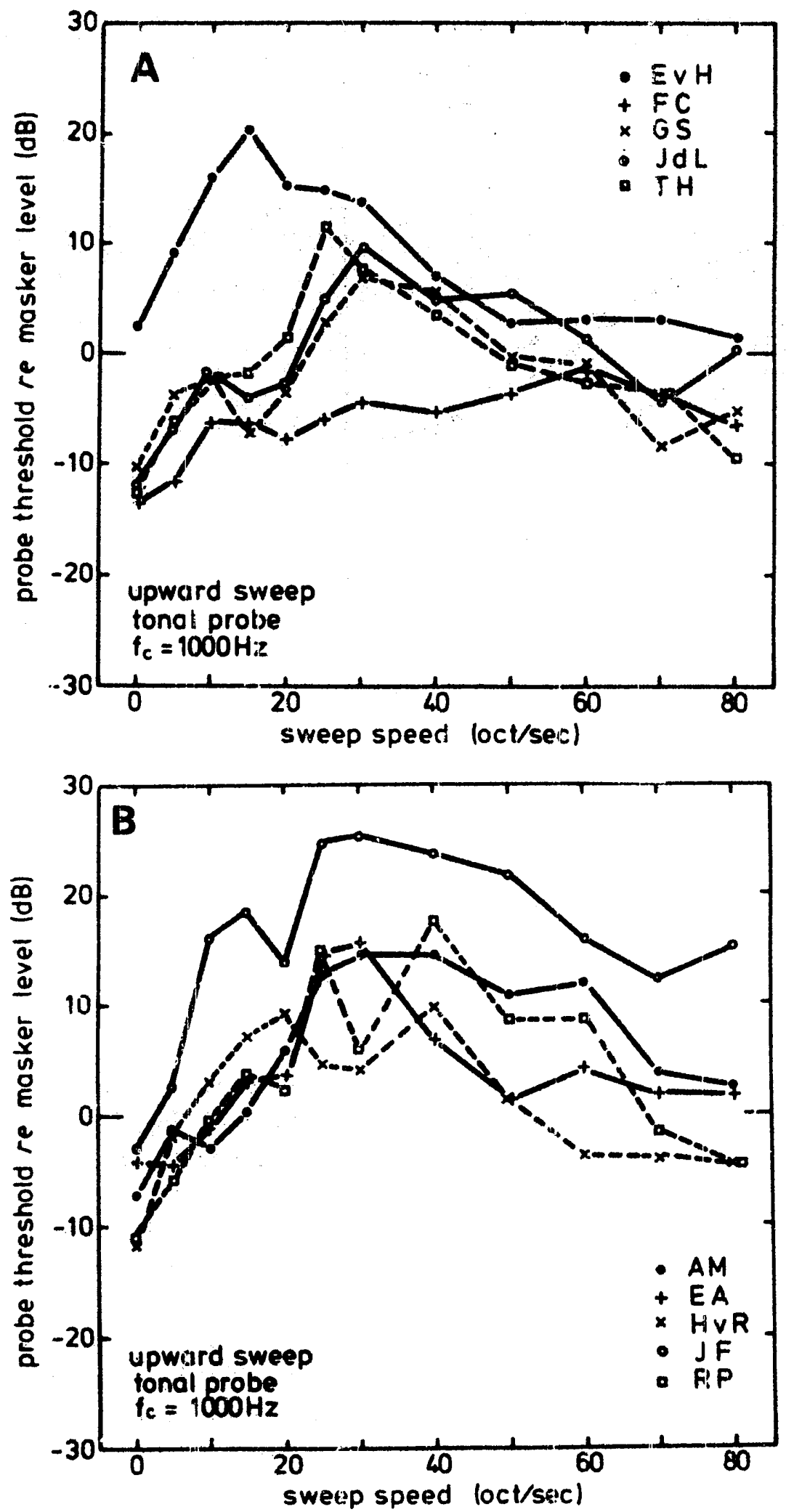

Fig. 3. Masked threshold as a function of sweep speed, $S$, for two groups (A and B) of five observers. The threshold level at which the probe is just detected is given in $\mathrm{dB}$ relative to the masker level. The masker was presented at constant SPL corresponding to $50 \mathrm{~dB} \mathrm{SL}$ for $S=0 \mathrm{oct} / \mathrm{s}$. Masked thresholds were measured for upward sweaps, tonal probes, a center firequency $f_{\mathrm{c}}=1000 \mathrm{~Hz}, 10 \mathrm{~ms}$ probe duration and $100 \mathrm{~ms}$ masker duration. The probe was added in-plase vith the masker. 


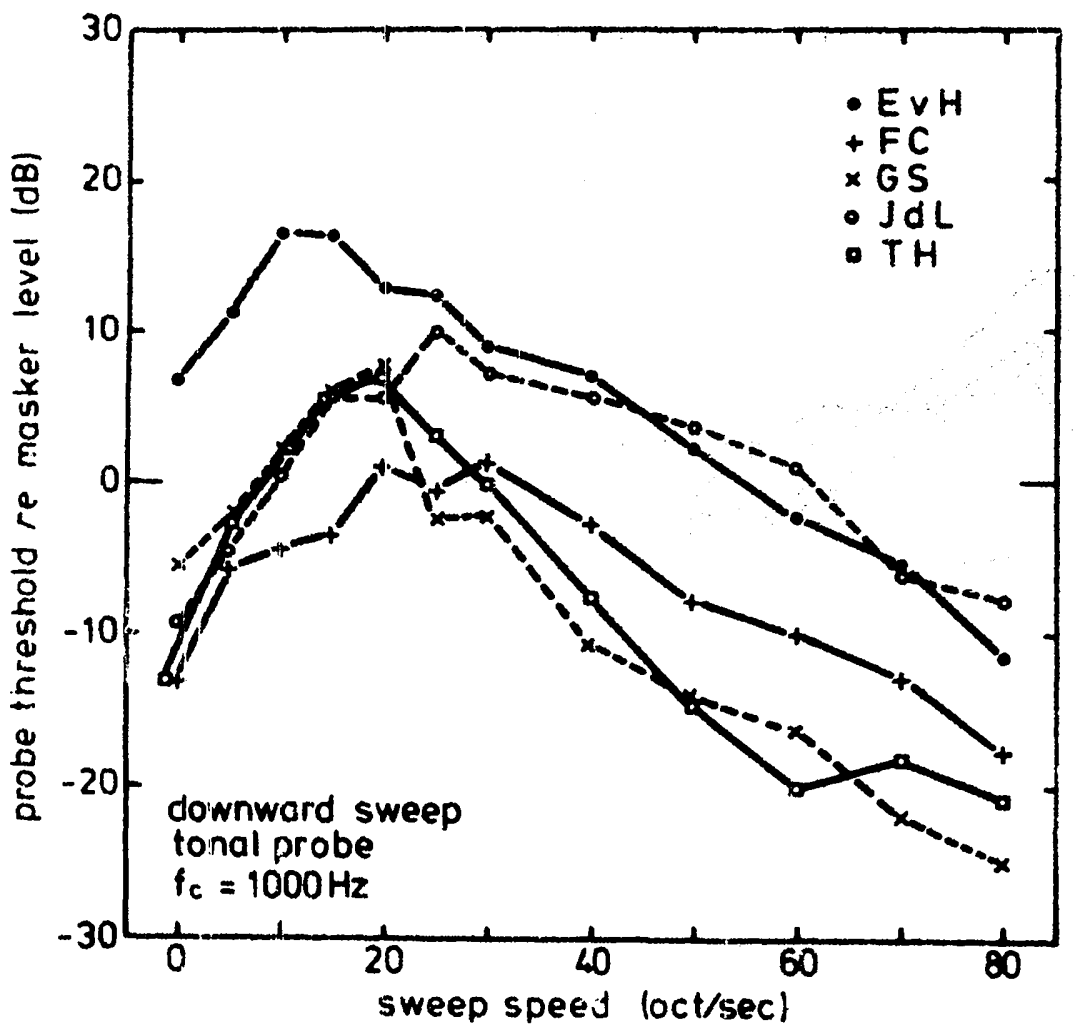

Fig. 4. As Fig. 3A for downward sweeps.

in-phase. The masker level was adjusted to $50 \mathrm{~dB}$ sensation level (SL) for each subject individually in the $S=0$ condition. The sound pressure level of the masker was not altered with other values of $S$ although the loudness did change (see Introduction). The masked threshold is expressed in the SPL of the probe at its maximum envelope value with respect to the SPL of the maske:

The results for upward sweeps are presented in Fig. $3 \mathbf{A}$ for five subjects and for five more subjects in Fig. 3B. The results for downward sweeps are presented in Fig. 4 for the five subjects in Fig. 3A. Plotted data are average values for two measurements carried out with about one month in between. The results may be summarized as follows:

1. All subjects show the same trend: with increasing sweep speed masked threshold first incr zases (the probe is detected at higher probe levels), then reaches a maximum, and finally drcreases at high speeds.

2. There appear to be significant inter-individual differences which in some conditions reach as much as $25 \mathrm{~dB}$. The inter-individual differences may be quantified by the coefficient of reliability, $r_{t t}$, which is defined as the proportion of true variance due to the inter-individual differences, or as unity minus the proportion of 'error' variance due to spread in adjustments per individual. It can be estimated from the correlation coefficieni, $r$, for the results of the first and second measurement (the test and the retest) by applying the formula of Spearman and Brown: $r_{t t}=2 r /(1+r)$ (cf. [5]). For upward sweeps we find $r_{\mathrm{tt}} \geqslant 0.90$ except for $S=0$ oct $/ \mathrm{s}$, where $r_{\mathrm{tt}}=0.83$, and for $30 \leqslant S \leqslant 60$ oct $/ \mathrm{s}$ where $0.70<r_{t t}<0.90$. For downward sweeps we find $r_{t t} \geqslant 0.90$ except at $S=30$ oct $/ \mathrm{s}$ where $r_{t t}=0.61$. 
3. For upward sweeps maximum rnasking occurs for ten subjects at an average value of $S=30 \mathrm{oct} / \mathrm{s}$ and the increase in masking with respect to the $S=0 \mathrm{oct} / \mathrm{s}$ condition averages $21 \mathrm{~dB}$.

4. For downward sweeps naximum masking occurs for five subjects at an averagge value of $S=20 \mathrm{oct} / \mathrm{s}$ and the increase in masking averages $15 \mathrm{~dB}$. For these five subjects maximum masking with upward sweeps occurs at $S=32 \mathrm{oct} / \mathrm{s}$ and the increase in masking is $19 \mathrm{~dB}$ (Fig. 3A).

5. A faster decrease of the masked thresholds at high sweep speeds $(S>50 \mathrm{oct} / \mathrm{s})$ is found for downward sweeps than for upward sweeps.

6. The average masked threshold for the $100 \mathrm{~ms}$ stationary masker $(S=0 \mathrm{oct} / \mathrm{s})$ and the in-phase $10 \mathrm{~ms}$ tonal probe is about $-8 \mathrm{~dB}$.

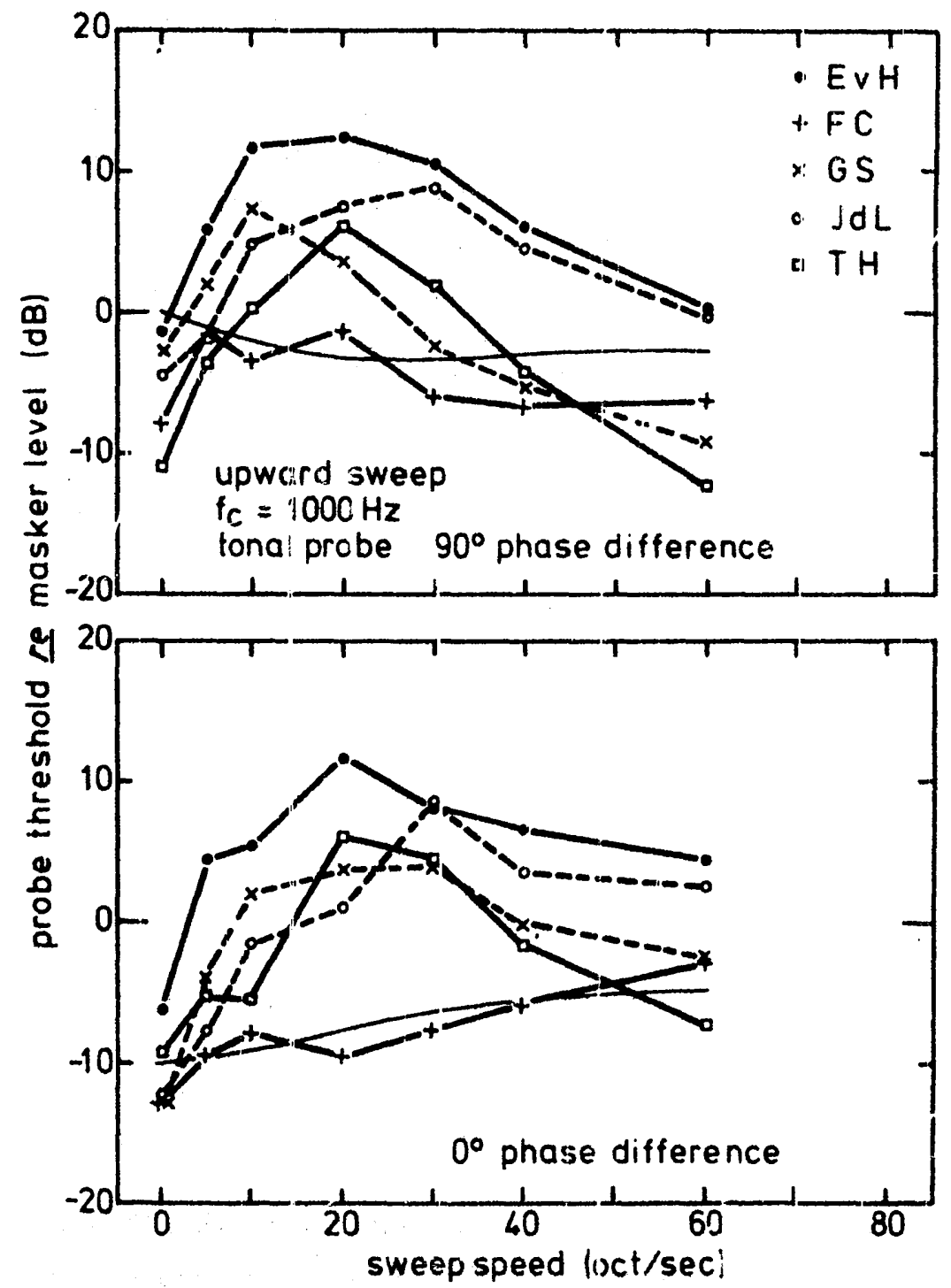

Fig. 5. As Fig. 3A for $90^{\circ}$. phase difference between probe and masker (upper panel) and for $0^{\circ}$ phase difference (lower parel). The masker was presented at $40 \mathrm{~dB} \mathrm{SL}(S=\uparrow$ oct $/ \mathrm{s})$. 


\section{Effect of phase on the masked threshold}

We mentioned in the Introduction that an increase of masked threshold for sweeping masker frequencies might be due, to some extent, to a running phase relation between the probe and the sweeping masker if the probe is presented in phase with the stationary masker (the $S=0$ oct/s condition). An increase of masked threshold for sweeping masker frequencies is not at all expected if the phase of the probe is shifted by $90^{\circ}$ with respect to the stationary masker. (The phase difference given for stationary maskers also holds for sweeping maskers in the common time center of probe and masker when the masker frequency equals the probe frequency.)

For the five subjects in Fig. 3A, masked thresholds were measured as a function of sweep speed with phase differences of $90^{\circ}$ and $0^{\circ}$. The results for $90^{\circ}$ phase difference are presented in the upper parel of Fig. 5 , the results for $0^{\circ}$ phase difference in the lower panel. Added to Fig. 5 as thin lines are predictions of the masked thresholds based on a $0.3 \mathrm{~dB}$ increment adding the probe, calculated over a $100 \mathrm{~ms}$ integration period and over the full sweep disregarding the critical band but taking into account the running phase relation.

In contrast with the prediction a pronounced increase of masked threshold is found for $90^{\circ}$ phase difference. Moreover, the increase of masked threshold for $0^{\circ}$ phase difference is much greater than predicted. Even the subject with the smallest effect (FC) shows an increase of masked threshold with respect to the $S=0 \mathrm{oct} / \mathrm{s}$ condition for $90^{\circ}$ phase difference and a greater increase than predicteơ for $0^{\circ}$ phase difference. These differences between experimental results and predictions do not depend critically on the chorice of the parameter values made in predicting the masked thresholds (see Discussion).

The results may be summarized as follows:

1. For $90^{\circ}$ phase difference maximum masking occurs at an average value of about $S=20 \mathrm{cct} / \mathrm{s}$ and the increase in masking averages $12 \mathrm{~dB}$. The average masked threshold fc: $t$... : s ationary masker $(S=0$ oct $/ \mathrm{s})$ i. $-5.3 \mathrm{~dB}$.

2. For $0^{\circ}$ phase difference maximum masking occurs at $S=32 \mathrm{oct} / \mathrm{s}$ and the increase in masking is $16 \mathrm{~dB}$. The corresponding values were $32 \mathrm{oct} / \mathrm{s}$ and $19 \mathrm{~dB}$, respectively, in the previous experiment at $50 \mathrm{~dB}$ SL (Fig. 3A). The masked threshold at $S=0 \mathrm{oct} / \mathrm{s}$ is $-10.7 \mathrm{~dB}$ (previously $-8.1 \mathrm{~dB}$ ).

\section{B. NOISE PROBE}

\section{Stimuli}

Only the differences with respect to the stimuli described in Part A will be mentioned here.

The noise probe was obtained by passing a burst of white noise with a Gaussian envelope through a 1/3-octave nilter (Brüel and Kjaer, type 1615). The center frequency of the $1 / 3$-octave was equal to the center frequency of the masker, 1 or $5 \mathrm{kHz}$.

The sweeping masker was produced by a voltage-controlled oscillator (Hewlett Packard 330.0 with 3305 A plug-in unit). This oscillator produces exponential frequency sweeps when linear voltage ramps are applied to the modulation input. The sweep speed could be controlled easily by the amplitude of the voltage ramp. 
In the experiments of Part B, onset and offiset of both the masker ard the probe were Gaussian-shaped. Rise and fall times of the masker were equal to $5 \mathrm{~ms}$, as defined from half-height to full-height of the one-sided Gaussian mnvelope. The 10- and 25-ms probe envelopes were fully Gaussian-shaped whereas the $40 \mathrm{~ms}$ probe consisted of a $5 \mathrm{~ms}$ onse, a $30 \mathrm{~ms}$ stationary period at full-height and a $5 \mathrm{~ms}$ offset. All probe and masker durations are defined from half-height at scimuius onset to half-height at stimulus off'set.

\section{Results}

The measuring procedure was the same as in the experiments of Part A. Data presented in Figs. 6-11 are average values of four measurements. All measurements were carried out at masker levels of $60 \mathrm{~dB}$ SPL.

\section{Masked thresholds for upward and downward sweeps}

Masked thresholds were measured as a function cf sweep speed up to about $S=115$ oct/s. Probe duration was $10 \mathrm{~ms}$ and masker duration $50 \mathrm{~ms}$. A masker cluration of $50 \mathrm{~ms}$ implies at the highest sweep speed of $S=115 \mathrm{oct} / \mathrm{s}$ a frequency sweep from 0.14 to 7.4 $\mathrm{kHz}$, with $1 \mathrm{kHz}$ as the center frequency. The result: for three subject; showr in Figs. 6 and 7 are qualitatively similar to those found for tonal probes (Figs. 3 and 4) but quantitatively there are differences which partly may be due to intersubject differences:

1. For upward sweeps (Fig. 6) maximum inasking occurs at an average value of $S=50$ oct/s (for tonal probes at $30 \mathrm{oct} / \mathrm{s}$ ) and the increase in masking with respect to the $S=0$ oct/s condition averages $12 \mathrm{~dB}$ (21 dB for tonal probe:s).

2. For downward sweeps (Fig. 7) maximum masking occurs at an average value of $S=$ $30 \mathrm{oct} / \mathrm{s}$ (for tonal probes at $20 \mathrm{oct} / \mathrm{s})$ and the increase in masking averages $11 \mathrm{~dB}(15 \mathrm{~dB}$ for tonal probes).

3. For stationary maskers the threshold averages $-15 \mathrm{~dB}(-8 \mathrm{~dB}$ for tonal probes).

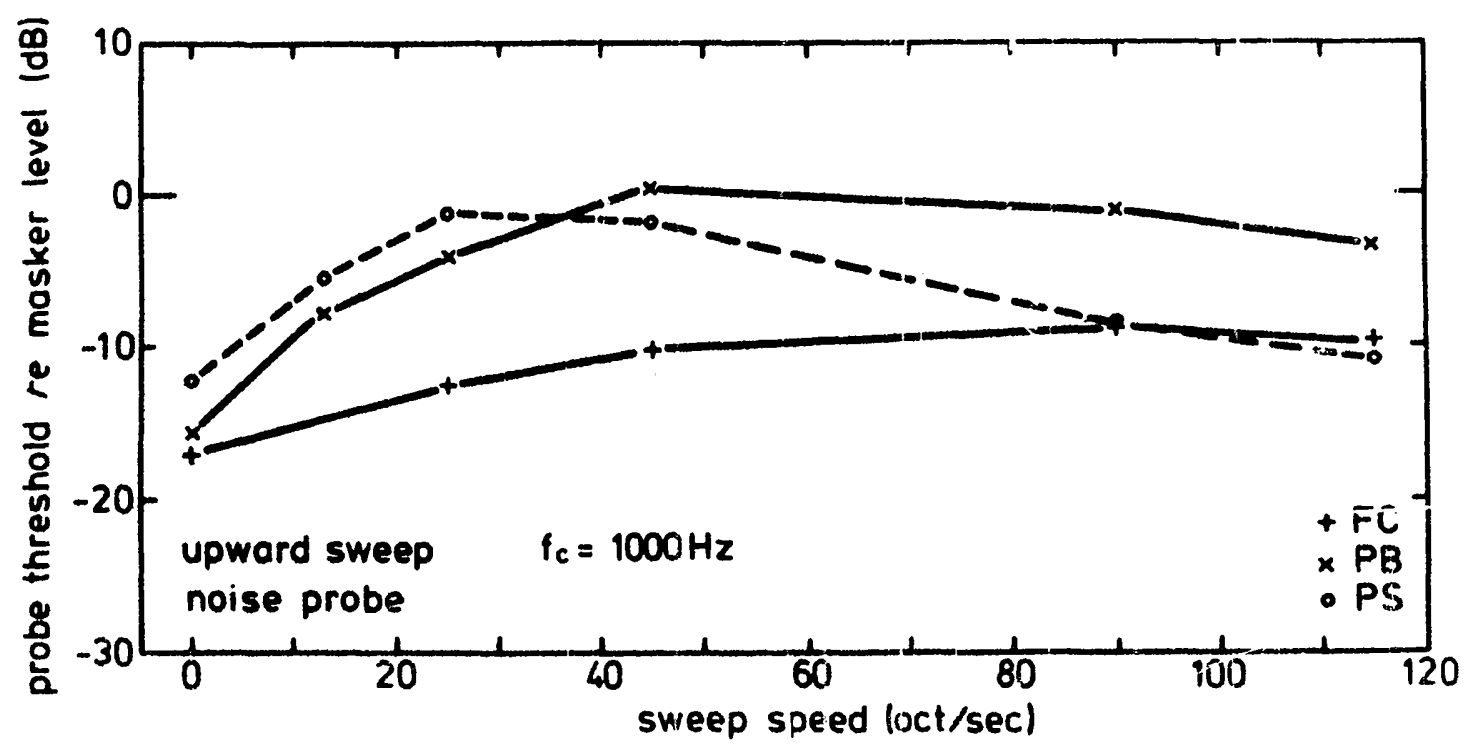

Fig. 6. Masked threshold as a function of sweep speed for noise probes and three subjects. Thresholds were measured for upward sweeps, $f_{c}=1000 \mathrm{~Hz}, 10 \mathrm{~ms}$ prote duration and $50 \mathrm{~ms}$ masker duration. 


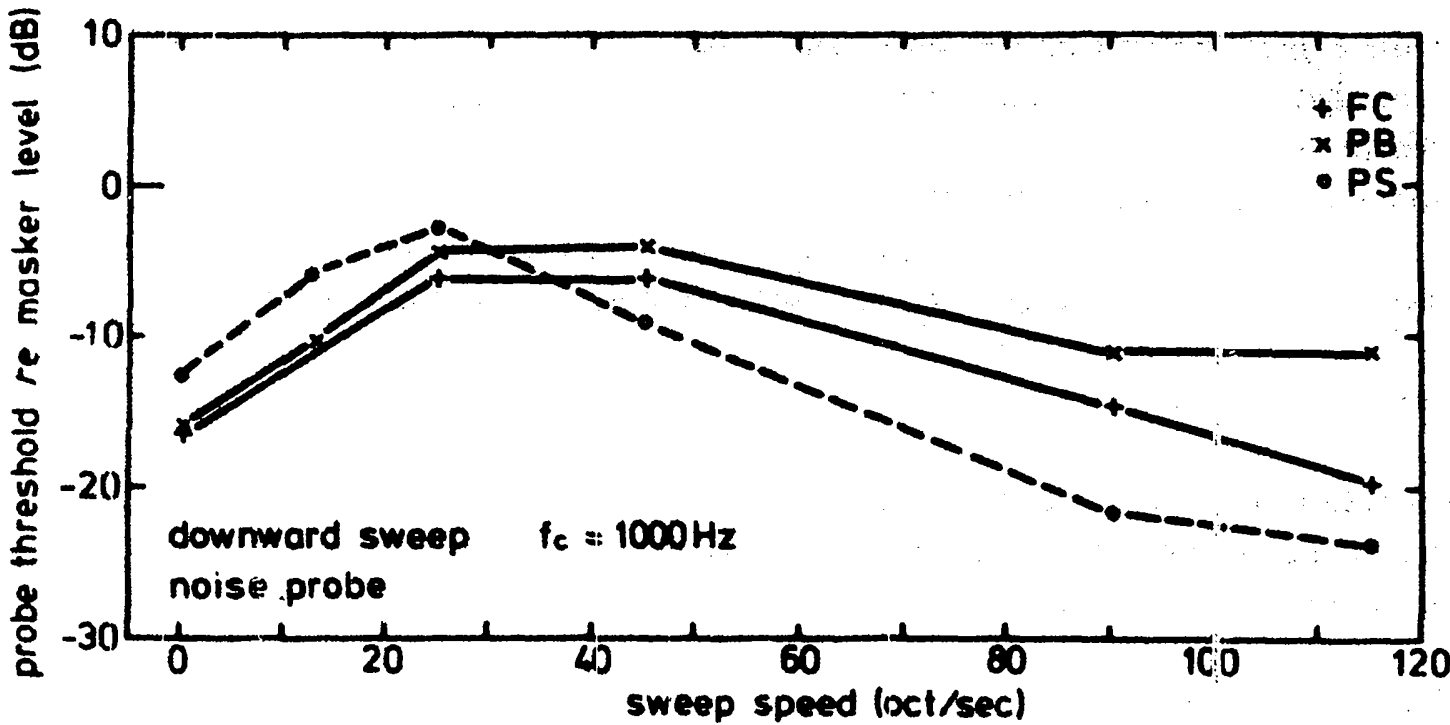

Fig. 7. As Fig. 6 for downward sweeps.
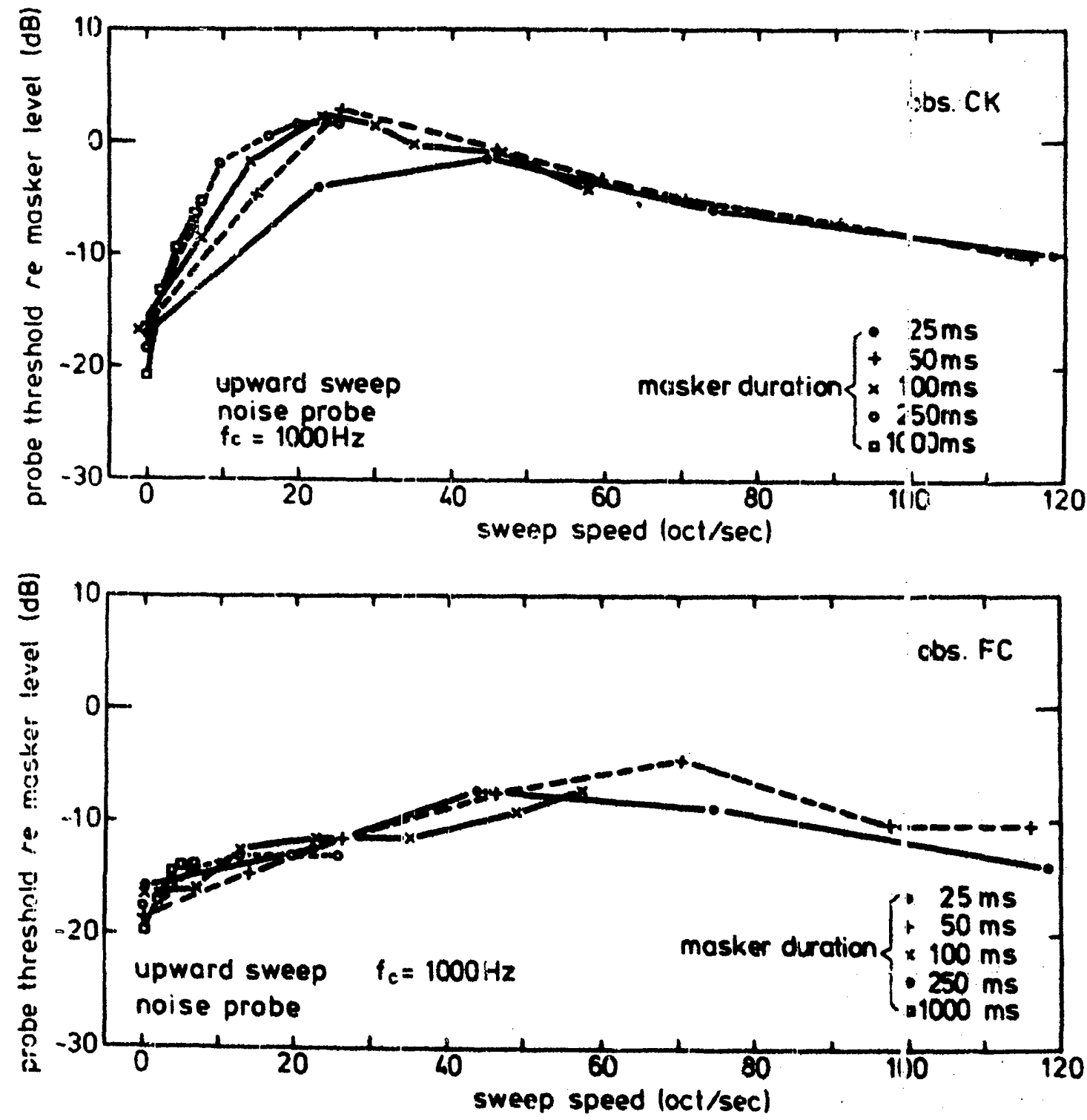

Fig. 8. Effect of masker duration nn masked threshold as a function of sweip sp:ed for two subjects. 

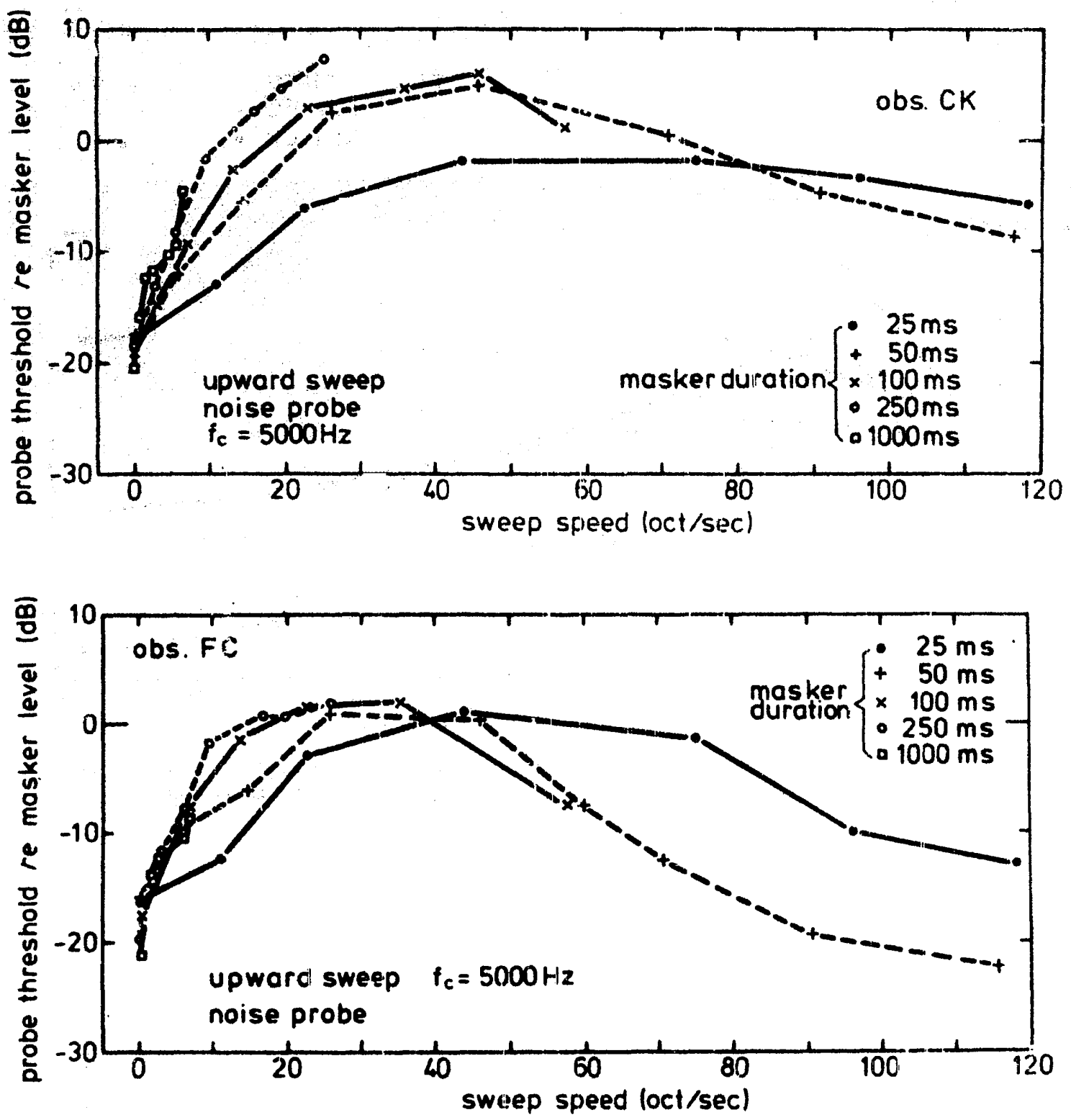

Fig. 9. As Fig. 8 but $f_{\mathrm{c}}=5000 \mathrm{~Hz}$.

4. As for tonal probes, important inter-individual differences were found. Also, at high sweep speeds a faster decay oi masked threshold for downward sweeps than for lipward sweeps was found again.

\section{Effect of masker duration on masked thresholl}

The effect of masker duration was measured for upward sweeps only. This choice of sweep direction was made arbitrarily. Probe duration was $10 \mathrm{~ms}$. The maximum sweep speed at which the masked threshold was measured depended on masker duration. We were not interested in high sweeps speeds at long masker durations because the range of the frequency sweep then lies outside the audible frequency range. Two subjects participated in this 


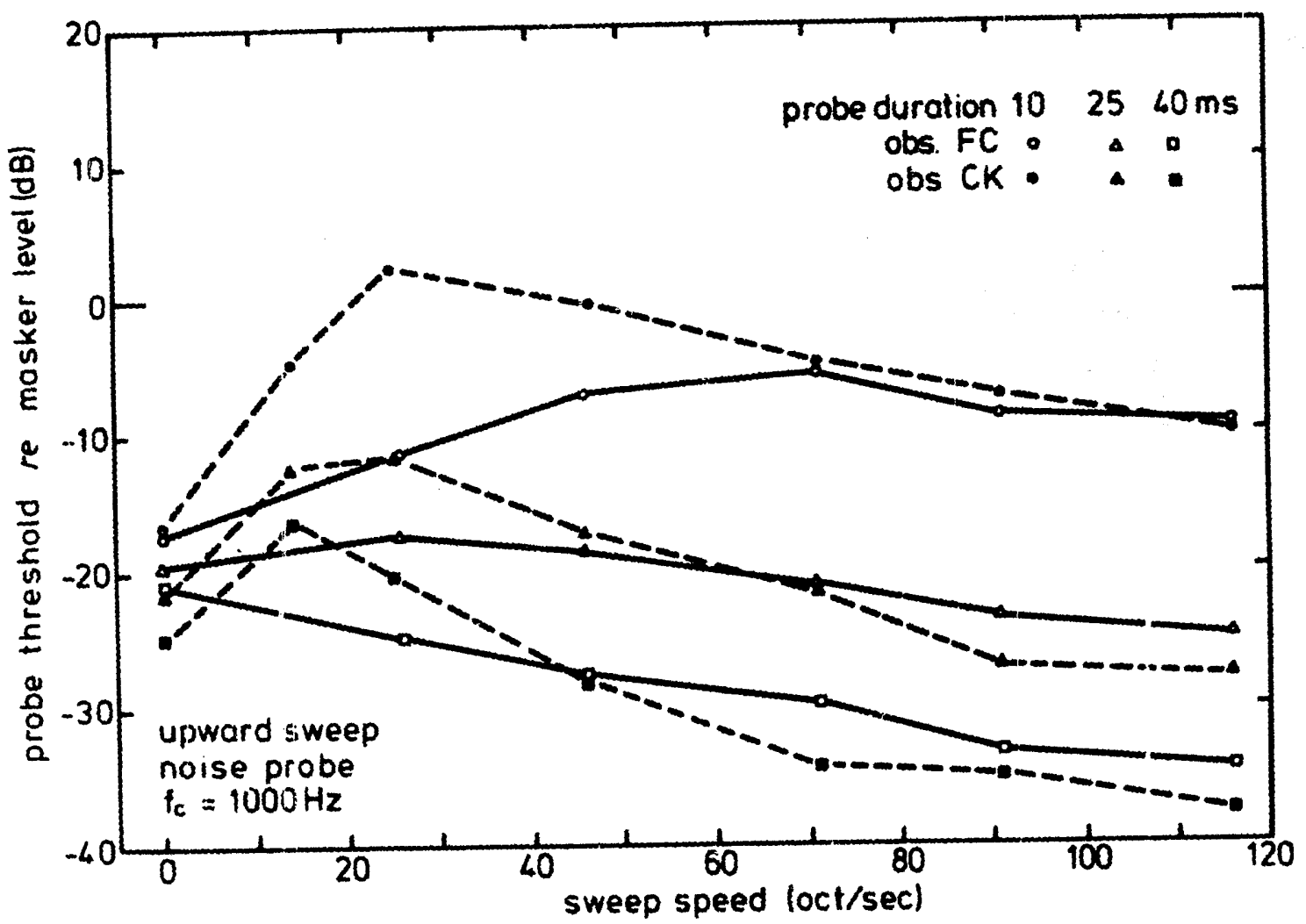

Fig. 10. Effect of probe duration on the masked threshold as a function of sweep speed for two subjects. Thresholds were measured for upward sweeps, noise probes, $f_{\mathrm{c}}=1000 \mathrm{~Hz}$ and $50 \mathrm{~ms}$ masker duration.

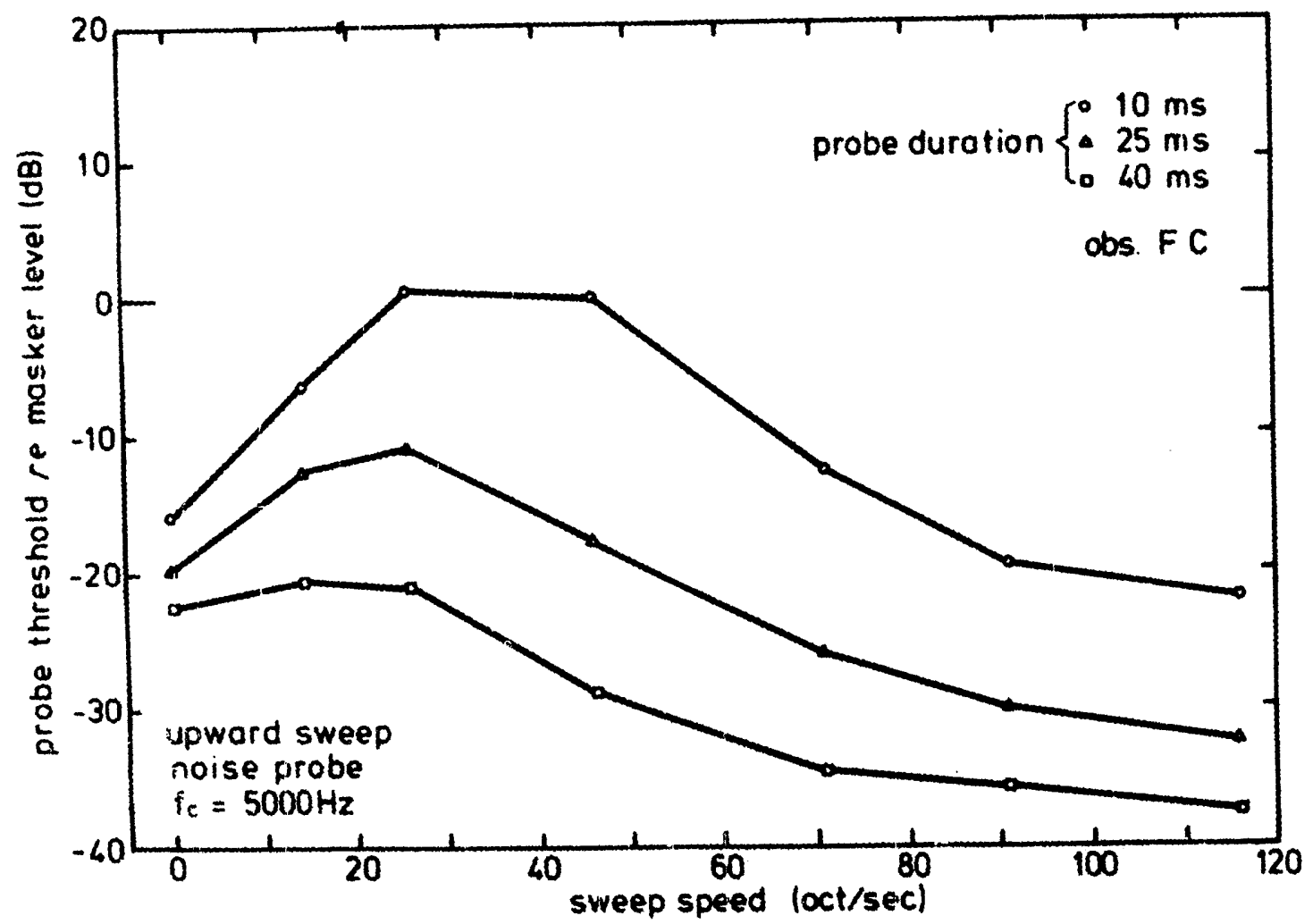

Fig. 11. As Fig. 10 for one subject and $f_{c}=5000 \mathrm{~Hz}$. 
frequency, respectively. They show that:

1. To a first approximation the masked threshold is determined by the sweep speed, irrespective of masker duration. With increasing sweep speed the trend of the curves again is an increasing masked threshold at low sweep speeds and a decreasing threshold at high speeds.

2. In addition the following effects may be distinguished: at $S=0 \mathrm{oct} / \mathrm{s}$ the masked threshold decreases with increasing masker duration; at small $S$ the threshold increases with masker duration.

3. Masked thresholds for center frequencies of 1 and $5 \mathrm{kHz}$ are similar for subject $\mathrm{C} \cdot \mathrm{K}$ but subject $\mathrm{FC}$ shows a less pronounced increase in masked threshold at $1 \mathrm{kHz}$ (also in Figs. 3A and 6).

\section{Effect of probe duration on masked threshold}

In this linal experiment masked threshold was measured again as a function of the sweep speed with masker duration fixed at $50 \mathrm{~ms}$ and probe duration the pàrameter. As mentioned before (Part B, Stimuli) the $40 \mathrm{~ms}$ probe envelope was not fuliy Gaussianshaped like the 10- and $25-\mathrm{ms}$ probes but it consisted of $5-\mathrm{ms}$ on- and offset times and a $30 \mathrm{~ms}$ period at full-height in between. This was done to avoid detection of the probe at its tails which for fully Gaussian envelopes might occur before detection at its top. The results are given in Fig. 10 for a center frequency of $1 \mathrm{kHz}$ and 2 subjects and in Fig. 11 for $5 \mathrm{kHz}$ and 1 subject. They show that:

1. A less pronounced maxinum in the threshold curves, or even no maximum at all, is found when probe duration is increased from 10 to $40 \mathrm{~ms}$. Further, the position of this maximum shifts to lower sweep speeds with increasing probe duration.

2. All masked thresholds decrease with increasing probe duration. This effect is greate: at high sweep speeds than at low speeds. Comparing tile thresholds found for 10 and 40 ras, a decrease by 8,3 and $6 \mathrm{~dB}$ (CK at $1 \mathrm{kHz}$ and $F C$ at 1 and $5 \mathrm{kHz}$, respectively) is found whereas these differences at $70<S<120$ oct/s are 28,24 and $18 \mathrm{~dB}$, respectively.

\section{DISCUSSION}

The main finding of the present experiments is an increase in masked threshold when the masker is swept up to certain sweep speeds, either upward or downward. This increase is found for probe durations up to $40 \mathrm{~ms}$, for tonal probes with $0^{\circ}$ and $90^{\circ}$ phase difference between probe and masker and for noise probes. The increase in masked threshold with respect to the $S=0$ ort/s condition is $21 \mathrm{~dB}$ (upward sweeps) and $15 \mathrm{~dB}$ (downward sweeps) for tonal probes with $0^{\circ}$ phase difference. The correspunding values are 12 and $11 \mathrm{~dB}$, respectively, foc noise probes. For tonal probes with $90^{\circ}$ phase difference the increase is $12 \mathrm{~dB}$ (upward sweeps only). A change of masker duration shows little effect on masked threshold: swesp speed appears to be ths important paramieter. Lastly, we found significant inter-individual differences between the masked thresho:ds.

We mentioned in the Introduction that the increase in masked threshold cannot be underst a probe sound is masked by a tone burst at the same frequency and hen the frequency of the tone burst is swept, we expect a decrease of masker energy in the critical band tuned 
to the probe sound and, hence, a decrease of masked threshold. The sweep speed at which this decrease is expected depends on critical bandwidth and integration time used in the model. The masker energy in the critical band will decrease when the masker passes through the critical band within the integration time. For example, if the critical bandwidth is set at $1 / 3$-octave and the integration time at $100 \mathrm{~ms}$ we expect a decrease at 3.3 oct/s. The results of several experiments $[4,11]$ suggest that the critical band broadens at short durations. Even if this were the case in our experiments, we would not expect an increase in masked threshold but a constant masked threshold up to higher sweep speeds followed by a decrease of masked threshold.

We also mentioned in the Introduction that for tonal probes the phase difference between probe and masker should be considered because the energy increment depends on this phase relation. Fo: stationary maskers the increment is maximal if the probe is added in-phase with the masker. When masker frequency is swept, this phase relation is preserved only in the common time center of probe and masker. Off time center, the sweeping frequency introduces phase shift which implies a decrease of the energy increment, and consequently, an increase of masked threshold because the same increment will be found at a higher probe level. For stationary maskers the energy increment is minimal if the phase difference between probe and masker is $90^{\circ}$. In that case no decrease of the energy increment, and thus no increase of masked threshold, is expected if masker frequency is swept. Nor is an increase of masked threshold expected for noise probes because the random phase relation implies that we may add the energies of masker and probe. In contrast with these predictions the results for tonal probes with $90^{\circ}$ phase difference and for noise probes showed a pronounced increase of masked threshold sweeping the masker frequency. Yet, a phase effect is present in the data of Fig. 5. The increase in masked threshold for $90^{\circ}$ phase difference (upper panel) and $0^{\circ}$ phase difference (lower panel) is 12 and $19 \mathrm{~dB}$, respectively, for the same group of five observes and upward swee ps.

In predicting the masked threshold (Fig. 5) we chose a $0.3 \mathrm{~dB}$ increment in order to match the $c$ ata for $S=0 \mathrm{oct} / \mathrm{s}$ and $0^{\circ}$ phase difference. This results in a prediction for $90^{\circ}$ phase difference that is higher than all data points. An increment of about $0.1 \mathrm{~dB}$ would have given a match with the data for $90^{\circ}$ phase difference but the prediction for $S=0 \mathrm{oct} / \mathrm{s}$ and $0^{\circ}$ phase difference would drop to about $-20 \mathrm{~dB}$. The choice of the increment value primarily affects the relative level of the masked thresholds and not so much the shape of the curves. Thus, the principal difference between experimental data and predictions, a pronounced increase of masked threshold with sweep speeds increasing from $S=0 \mathrm{oct} / \mathrm{s}$, is not critically affected by the choice of the increment value. The choice of iniegration time is even less important since it hardly affects the shapes of the predicted curves. The final choice made was to disregard the critical band. Including the critical band implies an additional decrease of the rnasked threshold aboire a cartain sweep speed. Further, we roughly expect a smaller effect of the phase difference between probe and masker on misked threshold.

A major point of concern in this study was how off-frequency detection of the probe might play a purt in these experiments. So far, we have assumed in our modelling that the probe is detected in the critical band tuned to the frequency common to probe and sta'ionary masker, the center frequency $f_{c}$. However, detection might tale place in critical 
bands tuned to slightly different frequencies. For example, the spectrum of the noise probe (Fig. 2) is wider than the spectrum of the stationary masker which, for durations greater than $10 \mathrm{~ms}$, will be narrower than the spectruin of the tonal probe in Fig. 2. This means that the probe-to-masker ratio at the edge frequencies of the noise probe $f_{\mathrm{c}} \pm$ $1 / 6-\mathrm{oct})$ is greater than at $f_{\mathrm{c}}$ and, thus, that the probe might be detected at lower levels in off-center-frequency critical bands. Whether or not off-frequency detection will occur also depends on the width and shape of the critical band filter, the absolute levels in the critical band filters ance temporal aspects involved in detection. Thus, predictions require a number of assumptions. Yet, some of our experimental data suggested the possibility of off-frequency detection. The masked threshold for noise probes and stationary maskers was very low, on average $-15 \mathrm{~dB}$. Moreover, these masked thresholds decreased with increasing masker durations (Figs. 8 and 9). This decrease might be due to al more narrow masker spectrum at longer durations. The two findings suggested the inclusion of experiments with tonal probes. The average masked threshold for tonal probes appeared to be higher, $-8 \mathrm{~dB}$ instead of $-15 \mathrm{~dB}$ for noise probes. This difference found for average values across different griups of subjects was also found for one subject (FC) who participated in both experimerts. Although a higher threshold was found for tonal probes, we are not quite sure that off-frequency detection does not occur for tonal probes. For sta" tionary maskers at $40 \mathrm{~dB}$ SL the effect of phase on masked threshold (Fig. 5) was not as great as predicted. One subjest (TH) showed no phase effect at all. The small phase effect might imply off-frequency detection since the phase relation is not preserved at the spurious frequencies (cf. $[6,12,13]$ ).

In order to study the effect of off-frequency detection on masked threshold we constructed a detection model. This model consisted of a number of staggered filters with high-fr :quency slopes of $100 \mathrm{~dB} / \mathrm{oct}$ and low-frequency slopes of $20 \mathrm{~dB} / \mathrm{oct}$-ver the first octave and $6 \mathrm{~dB} /$ oct at lower frequencies. Integration time was chosen at $30 \mathrm{~ms}$ and the detection criterion was a $1 \mathrm{~dB}$ increment in any one filter. Quiet threshold was simulated by internal noise. Predictions based on this model matched experimental data by Leshor itz and Wightman [6] very well. Leshowitz and Wightman determined the masked threshild for continous tonal maskers and 10-ms tonal probes with immediate onsets and offsets They found a less than proportional increase of masked threshold with increasing maske1 level. Moreover, at higher levels there was little effect of the phase difference between probe and masker on masked threshold. Leshowitz and Wightman attributed these $f$ ndings to off-frequency detection.

Having satisfied ourselves of a good match between our model predictions and experimental data on off-frequency detection, we applied the model to our sweep experiments. We tor $k$ the noise probes because these probes have the highest chance of being detected off-fre juency. The results showed no increase of masked threshold. With sweep speed increas ing from $S=0 \mathrm{oct} / \mathrm{s}$, the predicted masked threshold decreased immediately. The decrea $e$ for downward sweeps was greater than for upward sweeps. This difference was mainly due to off-frequency detection at lower frequencies than $f_{\mathrm{c}}$ for downward sweeps and al nost no off-frequency detection for upward sweeps. The fastcr decrease for downward $s$ weeps was found experimentally at high sweep speeds $(S \geqslant 5(\mathrm{oct} / \mathrm{s})$. We conclude that the increase in masked threshold is not due to off-frequency detection but that it is an inte resting phenomenon not yet understood. 
An increase in masked threshold was also noticed in results of an experiment reported by $Z$ wicker [16]. He measured the threshold of a short tonal probe masked by a continuously presented frequency modulated masker. For the stimulus conditions most comparable to our experiments we noticed an excessive masking of $10 \mathrm{~dB}$. This was not discussed in Zwicker's paper.

Increased responses to tones with a sweeping frequency are found in neurophysiological experiments; for example by $M \phi l l e r$ in rat cochlear nucleus $[7,8]$. However, we cannot decide from those data that this would mean a higher masked threshold. The increased response might imply an increased responsiveness to the probe as well as to the masker and, thus, a detectable increment in the response adding the probe might occur at the same probe-to-masker ratio.

\section{ACKNOWLE:DGEMENT}

We thank the subjects for participating in these experiments. This research was supported by the Netherlands Organization of the Advancement of Pure Research (ZWO).

\section{REFERENCES}

[1] Coninx, F. (1977): On the perception of changes in amplitude and frequency of acoustic signals. Dissertation, State University of Utrecht, The Netherlands.

[2] Coninx, F. and Smoorenburg, G.F. (1976): Loudness of stimuli with a sweeping frequency. Proc. Noise Control Conference, Warsaw, 149-152.

[3] Coninx, F. and Smoorenburg, G.F. (1979): Masking of short probe sounds by tone bursts with a sweeping frequency, J. Acoust. Sco. Am. 66, Suppl. 1, paper D6.

[4] Elliott, L.L. (1965): Changes in the simultaneous-masked threshold of brief tones. J. Acoust. Soc. Arn. 38, 738-746.

[5] Guilford, J.P. (1954): Psychometric Methods. McGraw-Hill, New York.

[6] Lesnowitz, B. and W/ightman, F. (1971): On-frequency masking with contiruous sinusoids. J. Accust. Soc. Am. 49, 1180-1190.

[7] M\$ller, A.R. (1971): Unit responses in the rat cochlear nucleus to tones of rapidly varying frequency and amplitude. Acta Physiol. Scand. 81, 540-556.

[8] Mфller, A.R. (1974): Coding of sounds with rapilly varying spectrum in the cochlear nucleus. J. Acoust. Soc. Am. 55, 631-640.

[9] Scharf, B. (1970): Critical Bands, in Foundations of Modern Auditory Theory, Vol. 1, pp. 159202. Euitor: G.V. Tobias. Academic Press, New York.

[10] Scharf, B. (1970): Loudness and frequency selectivity at short durations. In: Frequency Analysis and Periodicity Detection in Hearing, pp. 45.5-46,1. Editors: R. Plomp and G.F. Smoorenburg. Sijthoff, Leiden.

[11] Scholl, H. (1962): Das dyramischen Verhalten des Gehörs bei der Unterteilung des Schallspektrums in Frequenzgruppen. Aclistica 12, 101-107.

[12\} Vogten, L.L.M. (1978): Simultaneous pure tone masking: The dependence of masking asymmetries on interisity. J. Acoust. Soc. Am. 63, 1509-1519.

[13] Vogten, L.L.M. (1978): Low-level pure tone masking: A comparison of 'tuning curves' obtained with simultancous and forward masking. J. Acoust. Soc. Am. 63, 1520-1527.

114] Zwicker, E. and Feldtkeller, R. (1955): Über die Lautstärke von gleichförmigen Geräuschen. Acustica 5, 303-316.

[15] Zwicker, E. and Feldtkeller, R. (1967): Das Ohr als Nachrichtenempfänger, CHs. VI, XI. S. Hirzel Verlag, Stuttgart.

[16] Zwicker, E. (1974): Mithörschwellen und Erregungsmuster stark frequenzmodulierter Töne. Acustica 31, 243-256. 